\title{
COLOR HISTOGRAM EQUALIZATION THROUGH MESH DEFORMATION
}

\author{
Eric Pichon, Marc Niethammer* \\ Georgia Institute of Technology \\ \{eric, marcn\}@ece.gatech.edu
}

\author{
Guillermo Sapiro $^{\dagger}$ \\ University of Minnesota \\ guille@ece.umn.edu
}

\begin{abstract}
In this paper we propose an extension of grayscale histogram equalization for color images. For aesthetic reasons, previously proposed color histogram equalization techniques do not generate uniform color histograms. Our method will always generate an almost uniform color histogram thus making an optimal use of the color space. This is particularly interesting for pseudo-color scientific visualization. The method is based on deforming a mesh in color space to fit the existing histogram and then map it to a uniform histogram. It is a natural extension of grayscale histogram equalization and it can be applied to spatial and color space of any dimension.
\end{abstract}

\section{INTRODUCTION}

Contrast enhancement is a central problem of many image processing applications and Grayscale Histogram Equalization (GHE) is one of the simplest and most effective primitives for that purpose.

Given a grayscale image $I$, its histogram $H_{I}(i)$ is defined as the relative frequency of appearance of intensity $i$. In GHE (see [1,2]), the intensity $i=I(p)$ of every pixel $p$ is transformed to $j=J(p)=H_{I}^{C}(i)$ where $H_{I}^{C}=\int H_{I}$ is the cumulative histogram associated with $H_{I}$. The resulting image $J$ is equalized, by definition, its histogram $H_{J}$ is uniform which is equivalent to saying that all grayscale values are represented by the same number of pixels. This often results in spectacular subjective contrast enhancement (fig. 3(a) and 3(b)). Histogram specification, an extension of this technique, allows for any (not necessarily constant) user-supplied histogram.

Extending GHE to color images is not straightforward and various methods have been proposed to address this important problem.

The most simple extension is to apply GHE independently to the different bands of the color image. Another idea is to spread the histogram along its principal component axes [3] or along the brightness component of the image $[4,5]$. All these techniques only use marginal color histograms and therefore do not take into account the correlation between the different bands. In [6] a more interesting

\footnotetext{
${ }^{*} \mathrm{EP}$ and $\mathrm{MN}$ are supported by AFOSR, ARO, NSF, and HEL-MRI

${ }^{\dagger} \mathrm{GS}$ is supported by ONR, PECASE and NSF-CAREER.
}

approach is proposed that tries to extend the cumulative histogram to higher dimension.

If all these techniques increase the subjective contrast of color images, they all fail to produce a truly uniform color histogram. The main reason might be that contrarily to the grayscale case, imposing a uniform histogram for a color image results in the perception of severe color distortion. This perception can be explained (see [7]) by the difference between scotopic vision (monochromatic used mainly in varying low light conditions) and photopic vision (color used in standard lighting conditions). However, for many scientific and technical applications, aesthetic considerations are not prevalent. When visualizing pseudocolor images (multispectral satellite pictures for example), all available colors should be used.

\section{IDEA}

Given a vector-valued image $I=\left[I_{1}, \ldots, I_{n}\right]$, its histogram $H_{I}\left(i_{1}, \ldots, i_{n}\right): \mathbb{R}^{n} \rightarrow \mathbb{R}$ is defined as the relative frequency of occurrence of color $\left[i_{1}, \ldots, i_{n}\right]$. We want to create a transform $T: \mathbb{R}^{n} \rightarrow \mathbb{R}^{n}$ such that the image $J(x, y)=$ $T(I(x, y))$ is equalized i.e. $H_{J}\left(i_{1}, \ldots, i_{n}\right)$ is constant.

From now on, to simplify notations we will consider the case $n=2$. The images will be represented using red and green. Our results remain applicable to any higher dimensional color and/or spatial space.

We can see $T$ as a deformation of the color space $\mathbb{R}^{2}$. Therefore a good description for $T$ is the way it maps one mesh to another mesh. For example, imagine that we know a mesh $M_{I}$ which is mapped to a regular uniform mesh $M_{J}$ (fig. 1). It is then possible to construct a piecewise linear approximation $T_{P W}$ of $T$ by considering that all triangular half-cells of vertices $p_{I}, q_{I}$ and $r_{I}$ will be linearly mapped to the corresponding triangle of vertices $p_{J}=T\left(p_{I}\right), q_{J}=$ $T\left(q_{I}\right)$ and $r_{J}=T\left(r_{I}\right)$. Using the barycentric coordinates, $c_{I}=\alpha p_{I}+\beta q_{I}+\gamma r_{I}$ and we can define $T_{P W}$ by:

$$
T_{P W}\left(c_{I}\right)=\alpha p_{J}+\beta q_{J}+\gamma r_{J}
$$

Consider a pixel $(x, y)$ of the image. Its color is the vector $c_{I}=I(x, y)$. As $M_{I}$ is a mesh of the color space, $c_{I}$ is within a certain cell $K_{I}$. By definition of $M_{I}$ and $M_{J}$, all points $c_{I}$ of $K_{I}$ are mapped to points $c_{J}=T\left(c_{I}\right)$ of a cell $K_{J}=T\left(K_{I}\right)$ of $M_{J}$ through $T$. But as $J$ is an equalized 


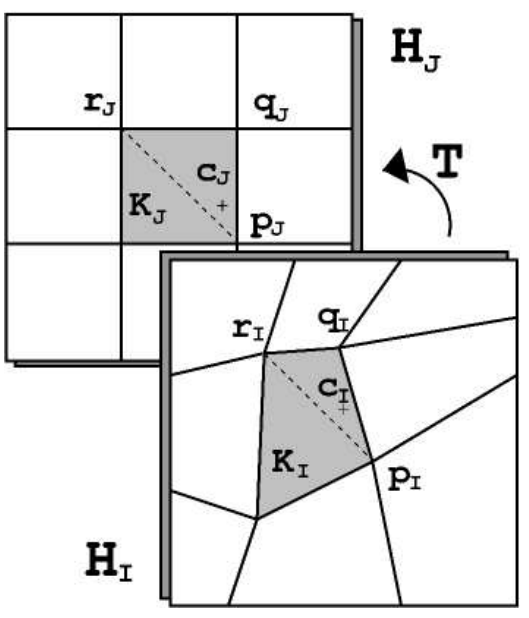

Fig. 1. The transformation $\mathrm{T}$ can be represented by a mesh

image, and $M_{J}$ is uniform, it follows that all cells of $M_{J}$ contain exactly the same number of points. Therefore this must also hold for $M_{I}$.

The only remaining difficulty is therefore to find a suitable mesh. Starting from a uniform $N \times N$ mesh, we can converge to a solution (fig. 2) by iteratively expanding all cells $K_{I}$ that do not contain enough points $\left(\frac{n_{X} n_{Y}}{N^{2}}-n_{K_{I}}>\right.$ 0 ) and contracting cells that already contain too many points $\left(\frac{n_{X} n_{Y}}{N^{2}}-n_{K_{I}}<0\right)$. This is done by moving the vertices $p_{I}, q_{I}, r_{I}$ of each cell $K_{I}$ towards the centroid $o_{K_{I}}$ or away from it. This leads to the following differential equation:

$$
\frac{\partial p_{I}}{\partial t}=\left(\frac{n_{X} n_{Y}}{N^{2}}-n_{K_{I}}\right)\left(p_{I}-o_{K_{I}}\right)
$$

where $n_{X}, n_{Y}$ are the dimensions of the image and $n_{K_{I}}$ is the number of pixels of the image whose colors are within cell $K_{I}$. At the steady state $n_{K_{I}}=\frac{n_{X} n_{Y}}{N^{2}}$ is constant, all cells contain the same number of points.

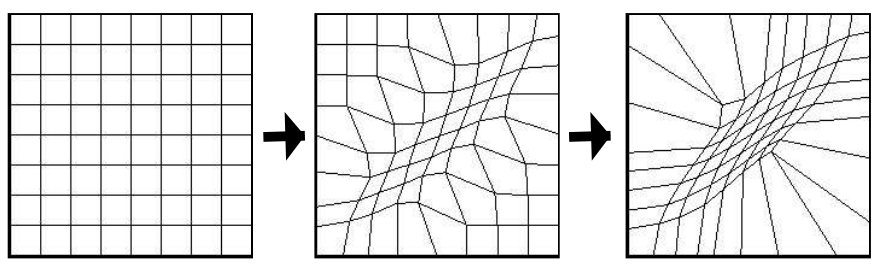

Fig. 2. Mesh deforming to fit histogram on figure 4(d)

\section{ALGORITHM}

Outline of the algorithm :

- determine the color histogram $H_{I}$ of the original image $I$.

- deform a mesh $M_{I}$ on the color space to fit the histogram $H_{I}$. (equation 2)
- use $M_{I}$ to define a piecewise linear deformation $T_{P W}$ of the color space. All cells of $M_{I}$ are linearly mapped to corresponding cells of the uniform mesh $M_{J}$. (equation 1)

- compute the equalized image $J=T_{P W}(I)$.

\section{RELATION WITH GRAYSCALE HISTOGRAM EQUALIZATION}

This formalism may seem extremely far from the simple "use the cumulative histogram approach" of GHE. We will now show that it is in fact a very natural extension.

Image 3(b) is the result of a GHE on image 3(a). To compute the output of our algorithm, we determine the histogram of the original image and then generate a mesh such that each cell (here each interval) contains the same number of pixels (fig. 3(c)). Colors of the original image are then piecewise linearly mapped to the same position in a corresponding uniform mesh. Therefore by construction, the histogram is equalized (fig. 3(d)).

Clearly the limits of the cells of this optimal mesh have to be $H^{C^{-1}}(k / N)$ with $k=0 \ldots N$ where $H^{C}$ is the cumulative histogram. Therefore, as shown on figure 3(e) the proposed equalization is a piecewise linear approximation of the grayscale histogram equalization (GHE) method.

\section{EXAMPLES}

In this section we will compare the result of traditional independent band GHE and the proposed color equalization on 2 pseudo-color images. We will see that the proposed transform equalizes almost ${ }^{1}$ perfectly the color histogram and thus makes an optimal use of all available colors.

Figure 4(e) is the original red-green image of the head of a moth as observed with an Environmental Scanning Electron Microscope. As we can see on the histogram (fig. 4(d) - for every color $(r, g)$ the probability of occurrence is mapped to the diameter of a disc), the red and green components of this pseudo-color image are strongly correlated ${ }^{2}$. Moreover, the marginal histograms $H_{R}$ and $H_{G}$ are already almost uniform. Therefore independent band GHE has almost no effect (fig. 4(c)) and the resulting histogram (fig. $4(a))$ is clearly not equalized.

On the contrary, the proposed color histogram equalization produces a much more colorful image (fig. 4(h)) and its histogram (fig. 4(f)) is almost ${ }^{1}$ perfectly uniform.

It is extremely instructive to consider the 2 meshes associated with these 2 transforms (fig. 4(b) and $4(\mathrm{~g})$ ). Note that even though independent band GHE does not explicitly use such an object, as a transform it can be represented by a

\footnotetext{
${ }^{1}$ rigorously, as in the case of GHE, the discrete nature of digital images does not necessarily allow for a perfectly constant histogram. For example the total number of pixels might not be divisible by the number of colors.

${ }^{2}$ This is not the original ESEM image. It was processed by the authors for the purpose of the demonstration.
} 


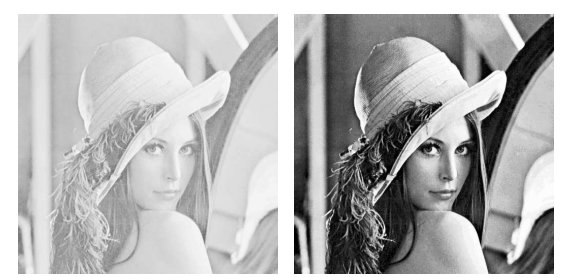

(a) Original image

(b) Grayscaleequalized image

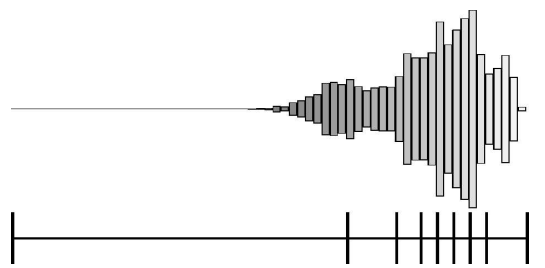

(c) Histogram of original image image and corresponding mesh

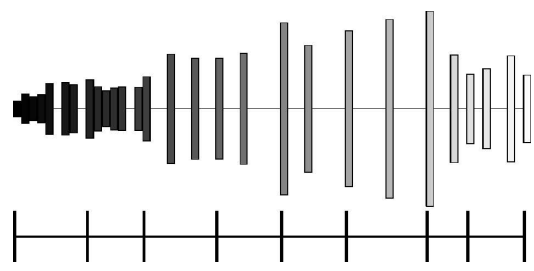

(d) Histogram of grayscale-equalized image and uniform mesh

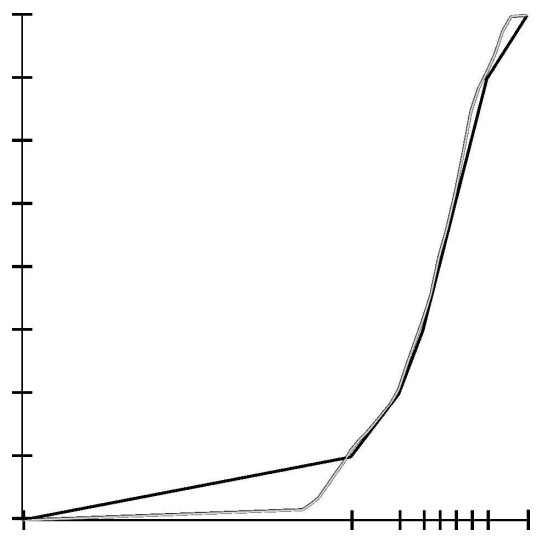

(e) Cumulative histogram (light gray) and proposed transform (black)

Fig. 3. Relation between proposed transform and grayscale histogram equalization mesh as discussed previously. As it operates independently on each band it should not surprise us that it is described by a separable mesh. In fact, if $H_{R}^{C}$ and $H_{B}^{C}$ are the red and green marginal cumulative histograms, vertical lines are defined by $H_{R}^{C^{-1}}(k / N)$ and horizontal lines by $H_{G}^{C^{-1}}(k / N)$ as discussed in 4 . As the marginal histograms are almost uniform, the cumulative marginal histograms (and their inverses) are close to the identity function $y=x$ and the resulting mesh is almost uniform.

On the contrary, the mesh of the proposed equalization is free from the separability constraint and makes an optimal use of the color space by fitting exactly the histogram of the input image.

When applied to a full RGB image (fig. 4(j)) the proposed algorithm (fig. 4(k)) clearly makes an optimal use of all available colors which leads to a much more vivid result than independent band GHE (fig. 4(i)).

\section{CONCLUSION}

In this paper we proposed an extension of grayscale histogram equalization to color images. This method can be applied to spatial and color spaces of any dimension. Contrarily to previous color histogram equalization methods, the resulting color histogram defined as a function $\mathbb{R}^{n} \rightarrow \mathbb{R}$ is constant which means that all colors are being used with the same frequency in the image.

As in grayscale histogram equalization this can be extended to histogram specification. Given two images $I$ and $K$, if $T_{I}$ and $T_{K}$ are the equalizing transforms then $T_{K}^{-1}$ 。 $T_{I}(I)$ will be a new version of $I$ with the same histogram as $K$.

\section{REFERENCES}

[1] A. K. Jain, Fundamentals of Digital Image Processing, Prentice Hall, 1989.

[2] R. E. Woods R. C. Gonzalez, Digital Image Processing, Prentice Hall, 2001.

[3] A.A. Schwartz J.M. Soha, "Multidimensional histogram normalization contrast enhancement," in Proc. 5th Canadian Symposium on Remote Sensing, 1978, pp. 86-93.

[4] I.M. Bockstein, "Color equalization method and its application to color image processing," J. Opt. Soc. Am., vol. 3, no. 5, pp. 735-737, 1986.

[5] W.F. McDonnel R.N. Strickland, C.S. Kim, "Digital color image enhancement based on the saturation component," Optical Engineering, vol. 26, no. 7, pp. 609-616, 1987.

[6] A.N. Venetsanopoulos P.E. Trahanias, "Color image enhancement through 3-D histogram equalization," in Proceedings, 11th International Conference on Image, Speech and Signal Analysis, 1992, vol. III, pp. 545-548.

[7] W. Livingston D.K. Lynch, Color and Light in Nature, Cambridge University Press, 2001. 


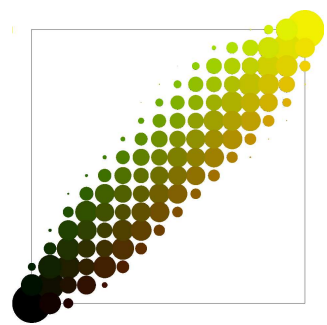

(a) Histogram of bandequalized image

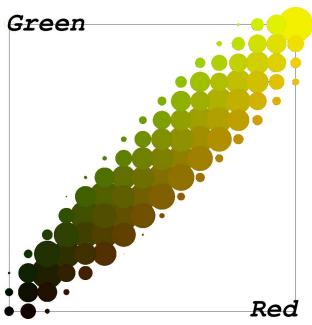

(d) Histogram of original image

$\therefore--i-0-0-0-0-1$ $\bullet \bullet \bullet \bullet \bullet \bullet \bullet \bullet \bullet \bullet$

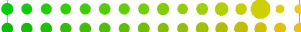

$\bullet \bullet \bullet \bullet \bullet \bullet \bullet \bullet \bullet \bullet \bullet \bullet \bullet \bullet ०$

ㄴ.

분

:

(1)

(

$\bullet \bullet \bullet \bullet \bullet$

:

:ำ:?:?:?:

(f) Histogram of colorequalized image

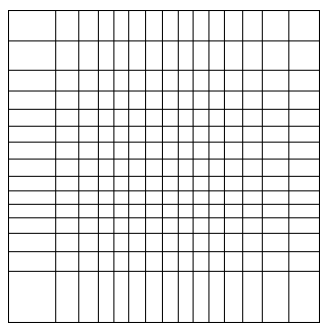

(b) Equivalent mesh of independent band grayscale histogram equalization

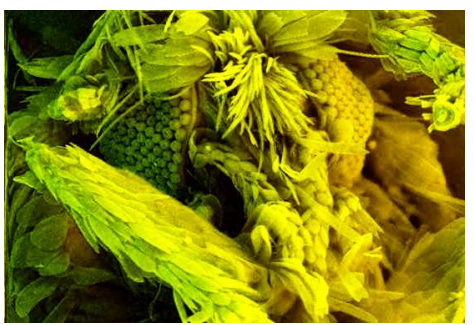

(c) Independent band-equalized image

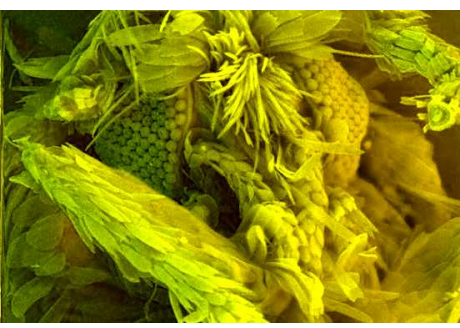

(e) Original RG image

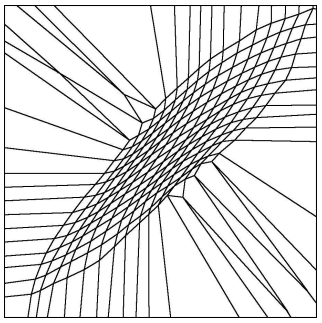

(g) Mesh of proposed transform

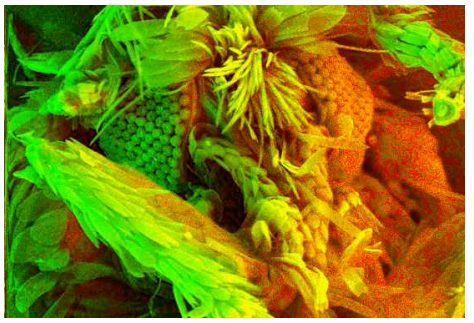

(h) Color-equalized image

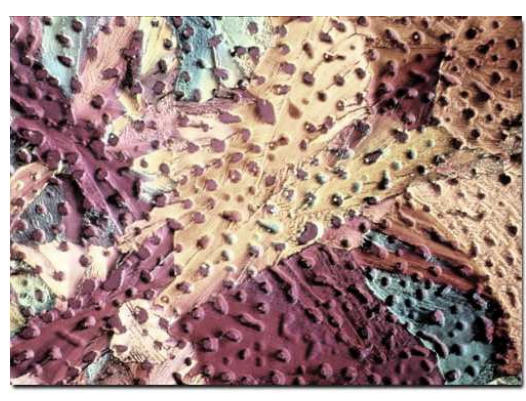

(i) Independent band grayscale histogram equalization

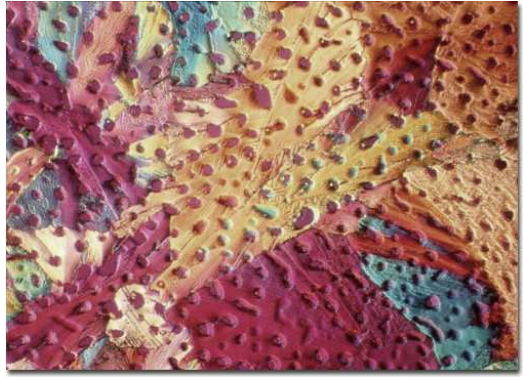

(j) Original RGB image

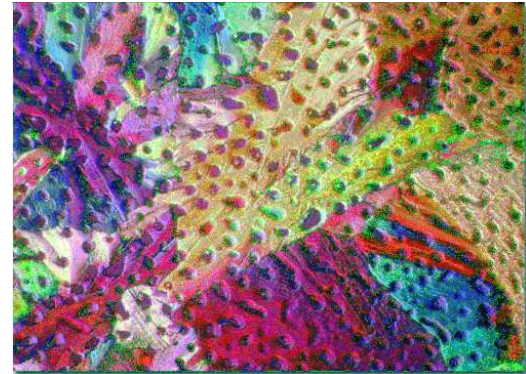

(k) Color-equalized image

Fig. 4. Examples. These are color images. 